\title{
Creation of Nanostructures on Polymer Surfaces Irradiated with Extreme Ultraviolet Pulses
}

\author{
A. Bartnik, H. Fiedorowicz, R. Jarocki, J. Kostecki, A. Szczurek and M. Szczurek \\ Institute of Optoelectronics, Military University of Technology, S. Kaliskiego 2, 00-908 Warsaw, Poland
}

A laser-plasma extreme ultraviolet source equipped with grazing incidence and Mo/Si collectors was used for surface modification of selected polymers. Surface morphology after irradiation was investigated. Different forms of nanostructures were obtained depending on polymer and irradiation conditions.

PACS numbers: 52.38.Ph, 82.50.Kx, 81.40.Wx

\section{Introduction}

Modification of surface topography of organic polymers accompanied by some chemical changes is very important in a wide spectrum of biotechnologies. Various surface structures in micro- and nanoscale were applied to study correlation between surface topography and cell adhesion [1-3]. Chemical modifications of polymers can be achieved using various chemical and biological methods, however, the most promising are physical methods such as ion implantation or UV irradiation in reactive atmosphere $[4,5]$. It seems that either physical or chemical surface modification can be also obtained using extreme UV (EUV) radiation. It could have some advantages connected with very short absorption length of EUV photons in any material. Such investigations were undertaken by authors of this paper using a laser-plasma EUV source based on a laser-irradiated gas puff target. Scanning electron microscopy (SEM) images of created nanostructures are presented.

\section{Laser-plasma EUV source}

In the experiments, a $10 \mathrm{~Hz}$ laser plasma EUV source based on a double stream gas puff target was used. The target was irradiated with $4 \mathrm{~ns} \mathrm{Nd:YAG} \mathrm{laser} \mathrm{pulses} \mathrm{with}$ energy $0.8 \mathrm{~J}$. Laser pulses were focused using a single spherical bi-convex lens with a focal length of $25 \mathrm{~mm}$. The target was created by pulsed injection of xenon or krypton into a hollow stream of helium with the use of an electromagnetic valve system equipped with a double nozzle setup. Detailed description of the source can be found elsewhere [6].

The laser-plasma EUV source emits radiation in a wide solid angle. It means that the radiation intensity drops as $1 / r^{2}$, where $r$ is the distance from the source. To obtain relatively high intensity at reasonable distance from plasma it is necessary to focus the radiation using a proper collector. Different kind of optics were developed that could serve as EUV collectors. The best are different kinds of grazing incidence or multilayer mirrors, however, polycapilary or Fresnel optics are also possible. In this experiment a grazing incidence and multilayer mirrors were employed. The grazing incidence collector manufactured in Reflex s r.o. is a part of a single ellipsoid coated with gold or molybdenium having a collection angle $0.75 \mathrm{sr}$. Roughness of its inner surface is better than $1 \mathrm{~nm}$. The grazing angle is about 15 degrees. It has a rotational symmetry and plasma is located in one of its focal points. The collector allows for effective focusing of radiation of $\mathrm{Kr}$ or Xe plasmas within the wavelength range $9-70 \mathrm{~nm}$. The maximum fluence is $65 \mathrm{~mJ} / \mathrm{cm}^{2}$ and FWHM of the focal spot is approximately $1 \mathrm{~mm}$. The grazing incidence mirror allows to obtain relatively high intensity in the focal spot but in a wide wavelength range. Another possibility offers an off-axis ellipsoidal multilayer mirror. Its $\mathrm{Mo} / \mathrm{Si}$ structure was optimized for relatively narrow spectral range $\lambda=13.5 \pm 0.5 \mathrm{~nm}$ under $45^{\circ}$ angle of incidence. Its collection angle is about $0.1 \mathrm{sr}$, so is much lower according to the collection angle of the grazing incidence mirror. On the other hand, size of the focal spot in this case is comparable to the plasma size, so is 2-3 times smaller comparing to the grazing incidence focal spot. An EUV fluence measured in the center of the focal spot was in this case $3 \mathrm{~mJ} / \mathrm{cm}^{2}$, an order of magnitude smaller in relation to the maximum fluence of EUV focused with the grazing incidence collector. Detailed description of the collectors can be found elsewhere [7]. To minimize the EUV absorption in a residual gas, filling the vacuum chamber during the source operation, collectors were mounted in an additional chamber and a differential pumping arrangement was applied.

\section{Experimental results}

The interaction experiments were performed with the use of $50 \mu \mathrm{m}$ polymer sheets from Goodfellow. The samples were used without any treatment. It 
was shown in our previous experiments that EUV fluence $>7 \mathrm{~mJ} / \mathrm{cm}^{2}$ is sufficient for efficient photoetching of polytetrafluoroethylene (PTFE), fluorinated ethylene-propylene (FEP) and poly(methyl methacrylate) (PMMA). In this experiment maximum fluence was $65 \mathrm{~mJ} / \mathrm{cm}^{2}$. It means that photo-etching and some surface changes of these polymers should occur. In a case of other polymers at least some surface modifications were expected. A polymer sample was mounted in a special holder on an $X Y Z$ translation stage. It was then possible to move it to different positions in respect of the focal point. The samples were irradiated with different number of pulses. For most of irradiated polymers exposure with 5 pulses was sufficient to see some surface modifications. Surface structures created in this case are small at the beginning with characteristic dimension hundreds of nanometers and are increasing with consecutive pulses. A good example of such polymer is polyethylene naphthalate (PEN). Structure images obtained in this polymer after irradiation with 1-10 pulses are presented in Fig. 1. It can be noticed that just after one pulse nanostructures with the characteristic size $200-300 \mathrm{~nm}$ are created. Five exposures result in $500 \mathrm{~nm}$ structures and after 10 exposures characteristic size of the structures exceeds $1 \mu \mathrm{m}$. Forms of the structures especially for 10 exposures suggest that they develop as a result of melting of a thin surface layer combined with releasing of volatile fractions of polymer chains. Similar structures were obtained for polystyrene (PS) and polyvinylidene chloride (PVDC). For larger number of exposures the structures increase up to several micrometers. In area of lower intensity the structures are similar but weaker.

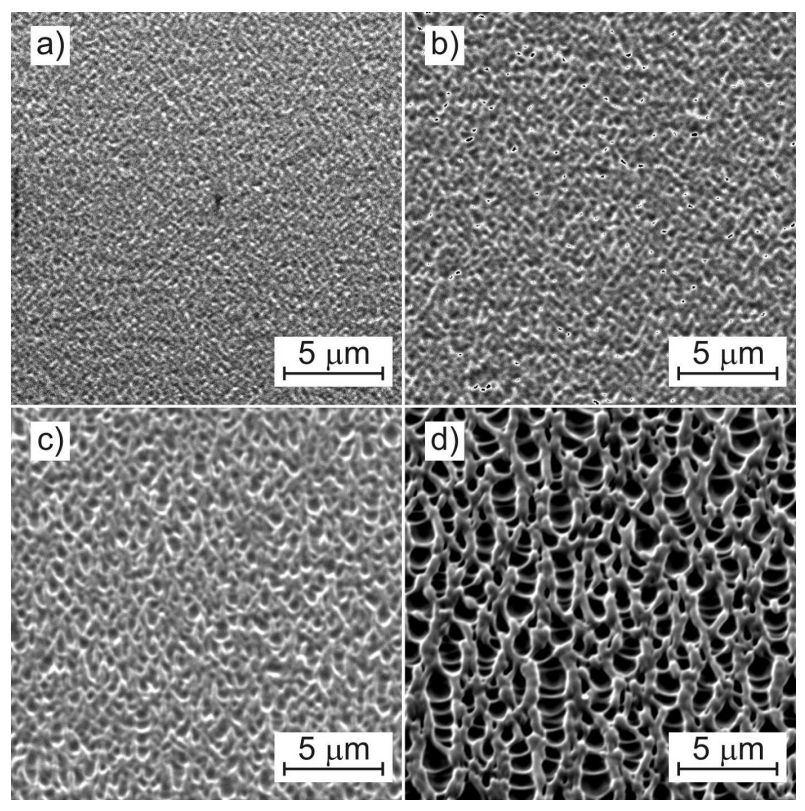

Fig. 1. Surface structures created in PEN for different numbers of EUV pulses: (a) 1 EUV pulse, (b) $3 \mathrm{EUV}$ pulses, (c) 5 EUV pulses, (d) 10 EUV pulses.
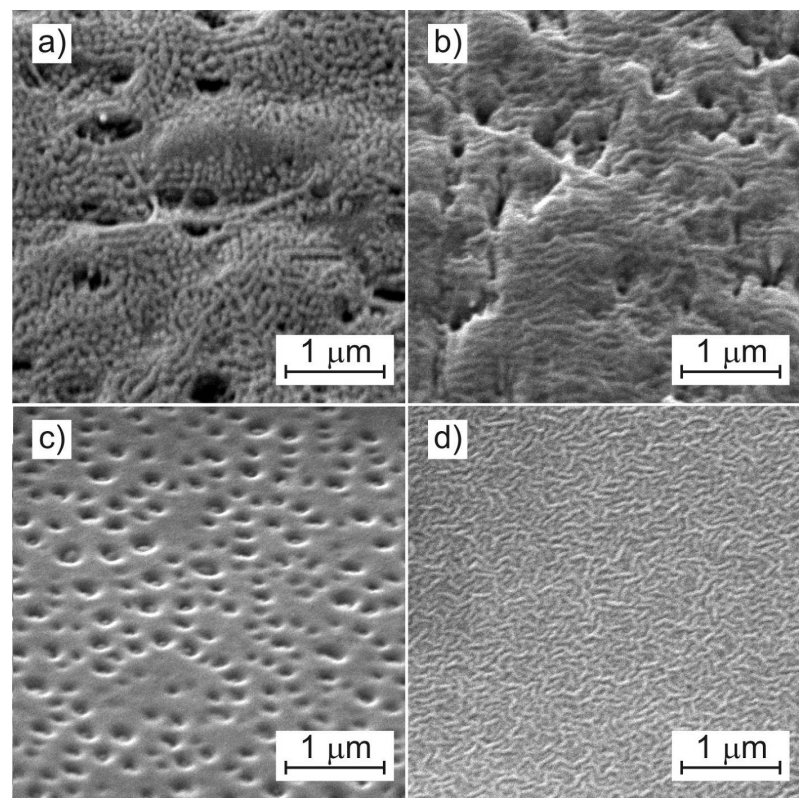

Fig. 2. Nanostructures created in PMMA and FEP using different collectors: (a) PTFE, grazing incidence collector, (b) PTFE, Mo/Si collector, (c) FEP, grazing incidence collector, (d) FEP, Mo/Si collector.

Another case was revealed for fluoropolymers like FEP or PTFE. In this case irradiation with relatively high fluence, close to the centre of the focal spot of the grazing incidence collector resulted in smooth ablation of a polymer material. Ablation rate exceeded $100 \mathrm{~nm}$ per pulse at the center. No surface structures similar to the structures created in PEN, PS or PVDC were obtained. An ablation mode was possible for relatively high EUV intensity in the area close to the center of the focal spot. For some distance from the center the intensity was not sufficient for smooth ablation. Also the Mo/Si collector could not assure such intensity. In these cases however significant surface modifications in nanoscale were created. The surface nanostructures obtained with the Mo coated grazing incidence collector have a form of nodules in PTFE (Fig. 2a) or pits in FEP (Fig. 2c) with the diameter of 100-150 nm. Irradiation with the Mo/Si collector created worm-like structures (Fig. 2b,d) with the smallest dimension of approximately $50 \mathrm{~nm}$. Nanostructures created this way are quite different than the structures obtained with the high fluence for PEN. Formation of the nanostructures shown in Fig. 2 can not be connected with melting because of relatively high melting temperature of the polymers and low EUV intensity. It was rather the result of preferential dry etching of amorphous polymer from a thin surface layer. Differences in structures obtained with the two different collectors were connected with significantly different spectral distributions of the focused radiation. It was checked that the worm-like structures can be created with the EUV radiation focused with the grazing incidence collector with 
additional Zr filter. In this case the EUV spectrum is limited to a relatively narrow wavelength range close to $10 \mathrm{~nm}$. It should be noticed that the $\mathrm{Mo} / \mathrm{Si}$ collector also reflects the EUV radiation in a narrow spectral band with maximum at $13.5 \mathrm{~nm}$. It means that forms of nanostructures resulting in EUV irradiation depend both on EUV fluence and spectral distribution. On the contrary to nanostructures created in PEN, PS or PVDC formation of such structures required irradiation with hundreds of EUV pulses.

\section{Conclusion}

In this paper we presented results of experiments concerning surface modification of organic polymers. The experiments were performed using the laser-plasma EUV source based on the double stream gas-puff target. The source was equipped with a grazing incidence and a $\mathrm{Mo} / \mathrm{Si}$ multilayer collectors. The grazing incidence collector allowed to focus EUV in a wide spectral range 9-70 $\mathrm{nm}$ while the $\mathrm{Mo} / \mathrm{Si}$ collector reflected radiation in a wavelength range $\lambda=13.5 \pm 0.5 \mathrm{~nm}$. Polymer samples were irradiated with different numbers of EUV pulses. Different kind of nanostructures were created depending on irradiation conditions and a kind of polymer.

\section{Acknowledgments}

The research was partially performed in the frame of the EUREKA project E! 3892 ModPolEUV and under
COST Action MP0601, funded by the Ministry of Science and Higher Education (decision No. 120/EUR/2007/02).

\section{References}

[1] Y. Wan, Y. Wang, Z. Liu, X. Qu, B. Han, J. Bei, S. Wang, Biomaterials 26, 4453 (2005).

[2] E.K.F. Yim, R.M. Reano, S.W. Pang, A.F. Yee, C.S. Chen, K.W. Leong, Biomaterials 26, 5405 (2005).

[3] E. Rebollar, I. Frischauf, M. Olbrich, T. Peterbauer, S. Hering, J. Preiner, P. Hinterdorfer, C. Romanin, J. Heitz, Biomaterials 29, 1796 (2008).

[4] T. Gumpenberger, J. Heitz, D. Bauerle, H. Kahr, I. Graz, C. Romanin, V. Svorcik, F. Leisch, Biomaterials 24, 5139 (2003).

[5] V. Svorcik, K. Rockova, E. Ratajova, J. Heitz, N. Huber, D. Bauerle, L. Bacakova, B. Dvorankova, V. Hnatowicz, Nucl. Instrum. Methods Phys. Res. B 217, 307 (2004).

[6] A. Bartnik, H. Fiedorowicz, R. Jarocki, J. Kostecki, J. Mikołajczyk, R. Rakowski, M. Szczurek, Proc. SPIE 5037, 389 (2003).

[7] A. Bartnik, H. Fiedorowicz, R. Jarocki, J. Kostecki, J. Mikołajczyk, R. Rakowski, M. Szczurek, Proc. SPIE 7361, 73610C (2009). 